\title{
The fascinating world of microorganisms
}

The discovery of microorganisms by Anton Van Leeuwenhoek (1632 - 1723) brought about a revolution in science. The immediate reaction to this discovery was confusion among the classical taxonomists at that time because all living things had been grouped either as plants or animals. It was not possible to include microorganisms in either group because they not only shared characters common to both but also possessed features unique to them and certain taxonomists called them "Chaos". With the accumulation of knowledge on their morphology, physiology, biochemistry and molecular biology it became evident that microorganisms are an extremely diverse group of organisms with only one feature in common, their minute size which make them invisible to the unaided eye. Today they are broadly classified into two domains Prokarya: the prokaryotic (non-nucleated) organisms that include the Archea, Eubacteria, Actinobacteria and Cyanobacteria and Eukarya accommodating the eukaryotic (nucleated) organisms including the microscopic algae, fungi and protozoa. Nevertheless the discovery of microorganisms led to a new area of science Microbiology.

Pioneering studies in microbiology were done by well known scientists mostly during the 19th century. Land mark studies in medical microbiology pioneered by Louis Pasteur, Ferdinand Cohen and Robert Koch (founders of bacteriology), the discovery of filterable viruses by Beijerinck and the work of many plant and animal pathologists which demonstrated that microorganisms are the causative organisms of contagious diseases, made them to be considered as dreaded germs and several methods and materials were developed to destroy them.

Meanwhile the unraveling of the crucial role played by microbes in fermentation, the discovery of biological nitrogen fixation, the isolation of penicillin followed by the production of several other antibiotics from microorganisms, the demonstration of the vital roles they play in the decomposition of organic matter for the functioning of nutrient cycles essential for the continuation of life on Earth, changed these attitudes. Subsequently the extensive use of microorganisms as handy tools to elucidate basic life processes such as photosynthesis, respiration, metabolism, genetics, molecular biology, modern biotechnology including genetic engineering and the recent use of microorganisms in the development of microbial-fuel cells had given them a special place in modern society. Today it is accepted that not more than $1 \%$ of all microorganisms are detrimental, a significant proportion being beneficial and a vast majority considered neutral, waiting to be explored and utilized.

The origin of life on Earth is believed to have commenced some 3.8 billion years ago (bya). Scientific evidence supports that during abiotic evolution the entities that gave rise to non-nucleated, prokaryotic microorganisms possessed only
Ribose Nucleic Acid (RNA) as their genetic material. The $\mathrm{O}_{2}$ evolving Protocyanobacteria appeared around 2.8 bya. During the Paleoproterozoic Era (2.5 to 1.6 bya) the Earth began and to cool followed by continuous torrential rain. These led to the proliferation of cyanobacteria in the marine phytoplankton which fixed and stored part of the carbon and released $\mathrm{O}_{2}$ that reduced atmospheric methane through oxidation. Meanwhile the chloroplasts that evolved through processes of endosymbioses between cyanobacteria and heterotrophic eukaryotic ancestors (Martin et al., 2002) accelerated $\mathrm{O}_{2}$ release to the atmosphere. Intense solar rays bombarding the earth converted some of the $\mathrm{O}_{2}$ to $\mathrm{O}_{3}$ which buildup a protective $\mathrm{O}_{3}$ layer that enabled the evolution and migration of life forms from the oceans to land. The oxygenation of the atmosphere also triggered off the development of aerobic respiration. Respiration was far more efficient than the anaerobic processes of fermentation and the aerobes proliferated at a dramatic pace driving the anaerobes to near extinction. Today aerobes are the dominant living forms among both flora and fauna thriving under an atmosphere containing $21 \%$ of oxygen. These evidences clearly show the crucial roles played by ancestral microorganisms and if not for them all other life forms that we see today may not be there.

Microorganisms are ubiquitous in their global distribution occupying a broad range of habitats including extreme ones such as hot springs, hypersaline localities, freezing environments and arid deserts. These versatile abilities and the intimate relationships including endosymbiosis they exhibit with all other forms of life are reflections of their pioneering ancestry. For several billion years they had been the only occupants of the primitive Earth. Recently Tripp et al. (2010) reported a globally distributed, periodically abundant $\mathrm{N}_{2}$-fixing marine, yet uncultivated, unnamed cyanobacterium UCYN-A, which was found to lack the oxygen-producing photosystem-II. They hypothesized it to possess a novel metabolism. Later UCYN-A was shown to have a symbiotic relationship with a eukaryotic, unicellular prymnesiophyte (Thompson et $a l ., 2012)$. This relationship between a cyanobacterium and a eukaryote similar to the one that gave rise to primitive chloroplasts, could perhaps be an indication of the existence of novel relationships among microbes yet to be discovered.

The widespread symbiotic relationships between microorganisms and all other living organisms on Earth had given rise to a novel theory of 'Symbiogenic Evolution' (Carrapico, 2015). This concept suggests that besides mutation, genetic recombination and natural selection, evolution would have involved symbiotic associations to form consortia of new structural dimensions. According to this theory most eukariyotic organisms are really consortia of organisms functioning in harmony towards common 
goals of development and adaptation. It has been reported that soil microorganisms seldom associate individually with higher plants but more so as consortia of microorganisms.

Found among the oligotrophic ocean phytoplankton is a little known cyanobacterium Prochlorococcus marinus which presents a unique case among the present day microorganisms. These vast populations are closely associated with numerous viruses that facilitate rapid lateral gene transfers among them and this endows upon them an intrinsic ability to display an array of physiological differences that provide an extraordinary stability to this heterogenomic community to internally adjust itself to the rapidly changing environmental conditions (Johnson et al., 2006).

From the foregoing it is evident that the 'Microbial World' presents an array of organisms with a very wide range of diversity and presents an invaluable gene pool which originated around 3.8 billion years ago. This germplasm had undergone evolution, natural selection and adaptation in harmony with all the changes undergone by the Earth. There is plenty more to discover among this fascinating bio-wealth and it is our foremost responsibility to protect this storehouse of invaluable genes for future utilization.

\section{S. A. Kulasooriya}

\section{REFERENCES}

Carrapico, F. (2015). Beyond neo-Darwinism. Building a Symbiogenic Theory of Evolution. Kairos Revista de
Filosophia and Ciencia 12: 47-53.

Johnson, I. Zackary, Zinser, Erik R., Coe, Allison, McNulty, Nathan P. E., Woodward, Malcolm S. and Chisholm, Sally M. (2006). Niche partitioning among Prochlorococcus ecotypes along ocean-scale environmental gradients. Science 311 (March): 1737 -1740 .

Martin, W., Rujan, T., Richly, E., Hamen, A., Cornelsen, S., Lin, T., Leister, D., Stoebe, B., Hasegawa, M. and Penny, D. (2002). Evolutionary analysis of Arabidopsis, cyanobacteria and chloroplast genomes reveals plastid phylogeny and thousands of cyanobacterial genes in the nucleus. Proceedings of the National Academy of Science, USA 99: 12246-12251.

Thompson A.W., Foster R.A., Krupke A., Carter B.J.,Musat N., Vaulot D., Kuypers M.M.M. and Zehr J.P. (2012). Unicellular cyanobacterium symbiotic with a single celled eukaryotic alga. Science 337: 1546-1550. DOI: http://dx.doi.org/10.1126/science.1222700110.

Tripp J.H., Bench S.R., Turk K.A., Foster R.A., Desany B.A., Niazi F., Affourtit J.P. \& Zehr J.P. (2010). Metabolic streamlining in an open-ocean nitrogen fixing cyanobacterium. Nature 464: 90-94. DOI: http:// dx.doi.org/10.1038/nature08786112. 